Cuffey, James. Photometry of NGC I907.

A preliminary estimate of the separation in space of the two galactic clusters M 38 and NGC I907 was made on the basis of revised and improved photo-red color indices. An attempt was then made to evaluate the mass of $\mathrm{M} 38$ from the conditions for stability of the companion cluster, NGC 1907, under the tidal action of M 38. The limiting diameter beyond which NGC 1907 can no longer be considered a stable cluster was estimated by star counts on long exposure photographs. The critical density for stability for NGC 1907 then led to an estimate of the mass of M 38 of the order of $6 \times \mathrm{IO}^{4}$ solar masses. Such a value was felt by the author to be much too high, for M 38 is not a very rich cluster. In fact, from star counts, and from the luminosity curve of $\mathrm{M} 38$, one would be apt to regard $5 \times 10^{3}$ solar masses as an upper limit to its mass.

A possible cause for the discrepancy may be found in the fact that the region of the clusters in Auriga is loaded with field stars belonging to the Auriga star cloud. The clusters, therefore, should not be regarded as isolated objects in space, but instead as parts of the Auriga star cloud. Under this condition, the stability criterion, derived by Hill, later by Bok, and by others, on the basis of the inverse square law of force does not apply to this particular problem. Loading of the cluster region with field stars may give an approach to uniform density in the region, and therefore the integrated law of force outward from M 38 may approach the inverse first power, and the method of mass estimation from stability considerations becomes completely insensitive. The presence of the field stars, however, does offer an explanation for the stability, under the shearing effects of galactic rotation, of the more widespread group of clusters, such as M 38, M 36, M 37, NGC 1907, NGC 1893, and others, in Auriga.

Kirdwood and Goethe Link Observatories, Indiana University, Bloomington, Ind.

\section{Donn, Bertram. On the interpretation of the Fraunhofer component of the solar corona.}

The discovery by Moore and Grotrian in 1934 of unwidened absorption lines in the spectrum of the outer corona together with observations of intensity distribution and polarization have led to the current view that the corona consists of two components. One has a continuous spectrum and arises from electron scattering. The second, which is of interest here, is the Fraunhofer component, containing the absorption lines. Previous workers have shown that the $\mathrm{F}$ component must be caused by scattering by solid particles, with diffraction as the dominant process.

The interpretation of the F component, therefore, provides an invaluable means of studying the nature of the interplanetary medium. If we describe the scattering cloud by a distribution law for radii, $a^{-p}$, space density law, $r^{-q}$, density of particles at I a.u., $N_{E}$, minimum and maximum radii, $a_{\min }$ and $a_{\max }$, and albedo, $\gamma$, the problem is to find the set of values for the parameters which will reproduce the observed coronal characteristics. As a result of the present general investigation, the available data do not seem sufficient to provide a definite answer and the program has turned to finding the restrictions which are placed on the possible solutions for the parameters.

A particular set of values which has been found to give fair agreement is $p=2, q=\mathrm{I}$, $a_{\max }=$ o.or. This is to be compared with Allen's result for the special case $a=\mathrm{IO}^{-3} \mathrm{~cm}$. for which $q=\mathrm{I}$ and Van de Hulst's solution for the case $q=0$, where $p=2.6$ and $a_{\max }>0.04 \mathrm{~cm}$.

Thus we can conclude that diffraction by a cloud of small particles will account for the F component, and reflection at large elongations will take care of the zodiacal light. However, the observations are too limited spatially and with respect to wave length to give a definite result for the properties of the cloud. This should be considered in any investigation requiring a knowledge of the properties of the interplanetary medium.

The possibility of extending the observations especially with infra-red sensitive photographic and photoelectric equipment holds out hope of obtaining a better description of the cloud of particles.

Harvard College Observatory,
Cambridge, Mass.

Duke, Douglas and K. Aa. Strand. The determination of the parallax and mass-ratio of 99 Herculis.

For the determination of the empirical massluminosity relation, it is essential to establish accurate individual masses of the components of selected double star systems. Under a joint program between the Yerkes and Dearborn Observatories the orbital motions as well as parallaxes and mass-ratios of several double star systems 